\title{
In adipose tissue, increased mitochondrial emission of reactive oxygen species is important for short-term high-fat diet-induced insulin resistance in mice
}

\author{
Sabina Paglialunga $\cdot$ Alison Ludzki • \\ Jared Root-McCaig • Graham P. Holloway
}

Received: 18 October 2014 / Accepted: 28 January 2015 / Published online: 11 March 2015

(C) Springer-Verlag Berlin Heidelberg 2015

\begin{abstract}
Aims/hypothesis Consuming a high-fat diet (HFD) induces insulin resistance in white adipose tissue (WAT) within 1 week. However, little is known about the initiating events. One potential mechanism that has remained largely unexplored is excessive mitochondrial emission of reactive oxygen species (ROS). Methods To determine the role of mitochondrial ROS emissions at the onset of insulin resistance, wild-type (WT) mice were placed on an HFD for 1 week. WAT insulin sensitivity and inflammation were assessed by western blot. In addition, we optimised/validated a method to determine ROS emissions in permeabilised WAT.

Results An HFD for 1 week resulted in impaired insulin signalling, increased c-Jun $\mathrm{NH}_{2}$-terminal kinase (JNK) phosphorylation and an increase in oxidative stress. These changes were associated with an increase in fatty-acid-mediated mitochondrial ROS emissions without any change in mitochondrial respiration/content. To determine that mitochondrial ROS causes insulin resistance, we used transgenic mice that express human catalase in mitochondria (MCAT) as a model of upregulated mitochondrial antioxidant enzyme capacity. MCAT mice displayed attenuated mitochondrial ROS emission, preserved insulin signalling and no inflammatory response following an HFD.

Conclusions/interpretation Findings from this study suggest that elevated mitochondrial ROS emission contributes to HFD-induced WAT insulin resistance.
\end{abstract}

Electronic supplementary material The online version of this article (doi:10.1007/s00125-015-3531-x) contains peer-reviewed but unedited supplementary material, which is available to authorised users.

S. Paglialunga $\cdot$ A. Ludzki $\cdot$ J. Root-McCaig $\cdot$ G. P. Holloway $(\bowtie)$ Department of Human Health and Nutritional Sciences, University of Guelph, 50 Stone Rd, Guelph, ON N1G 2W1, Canada

e-mail: ghollowa@uoguelph.ca
Keywords Insulin signalling $\cdot$ MCAT mice $\cdot$ Oxidative stress

$\begin{array}{ll}\text { Abbreviations } \\ \text { ANT } & \text { Adenine nucleotide translocase } \\ \text { DNP } & \text { 2,4-Dinitrophenol } \\ \text { ERK } & \text { Extracellular signalling-related kinase } \\ \text { ETF } & \text { Electron transfer flavoprotein } \\ \text { G3P } & \text { Glycerol 3-phosphate } \\ \text { GPDH } & \text { G3P dehydrogenase } \\ \text { HF(D) } & \text { High-fat (diet) } \\ \text { 4-HNE } & \text { trans-4-Hydroxy-2-nonenal } \\ \text { HSL } & \text { Hormone-sensitive lipase } \\ \text { ITT } & \text { Insulin tolerance test } \\ \text { JNK } & \text { c-Jun NH } \text {-terminal kinase } \\ \text { MAPK } & \text { Mitogen-activated protein kinase } \\ \text { MCAT } & \text { Transgenic mice that express human catalase in } \\ & \text { mitochondria } \\ \text { MCP-1 } & \text { Monocyte chemoattractant protein 1 } \\ \text { OXPHOS } & \text { Mitochondrial oxidative phosphorylation } \\ \text { ROS } & \text { Reactive oxygen species } \\ \text { SOD } & \text { Superoxide dismutase } \\ \text { SVC } & \text { Stromal vascular cells } \\ \text { WAT } & \text { White adipose tissue } \\ \text { WT } & \text { Wild-type }\end{array}$

\section{Introduction}

White adipose tissue (WAT), originally thought to be simply a lipid storage site, is now widely recognised as a dynamic organ involved in energy metabolism. WAT dysfunction may represent a significant mechanism in the rapid onset of whole-body glucose intolerance with high-fat (HF) feeding, as it has been estimated that adipose tissue can contribute up to 
$20 \%$ of whole-body glucose homeostasis $[1,2]$. Short-term (3-7 days) HF feeding results in an increase in WAT insulin resistance and hypoxia [3], as well as an upregulation of the expression of genes encoding inflammatory markers [4]. Even at this early time point, some [4], but not all, studies $[5,6]$ show WAT macrophage infiltration. As consumption of an HF diet (HFD) continues, WAT is typically characterised by elevated basal lipolytic activity [7], overt macrophage infiltration [8], upregulation of proinflammatory cytokines [9] and an accumulation of oxidative stress markers [10].

A recent study demonstrated that mitochondrial emission of reactive oxygen species (ROS) and oxidative stress markers were increased in subcutaneous WAT obtained from obese individuals with type 2 diabetes compared with control participants [11], suggesting alterations within mitochondria contribute to WAT insulin resistance. However, it is unclear if the observed increase in mitochondrial ROS emission was a cause or a consequence of WAT insulin resistance, and therefore the mechanistic link between mitochondrial ROS and the induction of WAT insulin resistance remains ambiguous.

It has also been suggested that adenine nucleotide translocase (ANT)-mediated mitochondrial uncoupling results in WAT hypoxia during an HFD, thereby inducing inflammation and insulin resistance [3]. However, in stark contrast, mechanisms that induce mild mitochondrial uncoupling, such as targeted upregulation of uncoupling protein 1 , genetic approaches that result in 'browning' of WAT and the administration of artificial mitochondrial uncouplers (i.e. 2,4-dinitrophenol [DNP]), improve insulin sensitivity and decrease obesity [12-14]. Mitochondrial uncoupling promotes energy dissipation in the form of heat loss, as the mitochondrial proton gradient is dissociated from ATP production. By lowering the proton gradient, mitochondrial uncoupling decreases ROS production [15]. In a feedback system an excessive amount of mitochondrial ROS emissions have been shown to induce mitochondrial depolarisation [16]. Therefore, the previously observed HFD-induced uncoupling may be a result of the HFD rather than a direct cause of insulin resistance.

The current study aimed to determine the mechanistic role of mitochondrial ROS in the initiation of HFDinduced insulin resistance in WAT. To this end, WT mice were fed chow or an HFD for 1 week and we evaluated markers of insulin signalling and inflammation in addition to directly measuring WAT mitochondrial ROS emissions. Furthermore, we used MCAT mice, which have the catalase antioxidant enzyme within mitochondria [17-19], to delineate a causal mechanism. Together, the current data implicate increased mitochondrial ROS in the acute HFD-induced activation of WAT insulin resistance.

\section{Methods}

Mice C57BL/6 male ( $n=11-12$; own colony) and WT and MCAT (B6.Cg-Tg(CAG-OTC/CAT)4033Prab/J; 016197) mice on the same C57BL/6 background ( $n=5-7$; Jackson Laboratories, Bar Harbor, ME, USA) at the age of 15 weeks were placed on either a chow diet $(10 \%$ energy from fat; Harlan, Indianapolis, IN, USA) or an HFD (60\% energy from fat; D12492, Research Diets, New Brunswick, NJ, USA) for 1 week. Animals had ad libitum access to food and water. Mice were housed in a barrier facility with a $12 \mathrm{~h} \mathrm{light/dark}$ cycle. All protocols were approved by and performed in accordance with the Committee on Animal Care guidelines at the University of Guelph.

Serum assays After 6 days of chow or HFD, an insulin tolerance test (ITT) was performed in awake mice as previously described [20]. Briefly, mice were fasted for $4 \mathrm{~h}$, starting at 06:00 hours, venous tail blood glucose was measured at $t=0$, $5,10,15,30,60,90 \mathrm{~min}$ after an i. p. insulin injection $(1 \mathrm{U} / \mathrm{kg}$ body weight; Humulin, Lilly, Indianapolis, IN, USA). Insulin (Millipore, Billerica, MA, USA), NEFA (Wako Chemicals, Richmond, VA, USA) and glycerol were measured, using commercially available kits, in $2 \mathrm{~h}$ fasted serum samples from anaesthetised ( $40 \mathrm{mg} / \mathrm{g}$ pentobarbitol) mice after 7 days of the diet intervention.

In vivo insulin stimulation WAT was excised before (basal) and $15 \mathrm{~min}$ after an intravenous insulin injection (1 U/kg body weight, Humulin) in anaesthetised mice. Tissue was immediately flash frozen in liquid nitrogen for western blot analysis, and the animals were then killed. Blood glucose was measured with a glucose meter (Abbott Diabetes Care, Alameda, CA, USA).

Western blot analysis WAT was homogenised as previously described [21]. Standard SDS-PAGE procedures were followed. Antibodies included total and phosphorylated Akt, c-Jun $\mathrm{NH}_{2}$-terminal kinase (JNK)1/2, P38-mitogen-activated protein kinase (MAPK), extracellular signalling-related kinase (ERK)1/2 and hormone-sensitive lipase (HSL; Cell Signaling, Danvers, MA, USA), as well as mitochondrial oxidative phosphorylation complexes (OXPHOS; MitoSciences, Eugene, OR, USA), monocyte chemoattractant protein 1 (MCP-1; Cell Signaling), catalase, superoxide dismutase (SOD)2 (Abcam, Cambridge, UK) and trans-4-hydroxy-2-nonenal (4-HNE; Alpha Diagnostics, San Antonio, TX, USA), with $\alpha$-tubulin (Abcam) as a loading control. Protein carbonylation (Oxyblot, Millipore) was determined according to the manufacturer's instructions.

Adipocyte cell sizing and immunohistochemistry Adipose tissue was fixed, embedded and stained as previously 
reported [22]. Macrophage infiltration was assessed by F4/80 (1:100, AbD Serotec, BioRad, Hercules, CA, USA) staining visualised using the Vectastain $\mathrm{ABC}$ kit (Vector Laboratories, Burlingame, CA, USA). Images were taken with an Olympus (Tokyo, Japan) microscope at $\times 40$ magnification and analysed with ImageJ 1.48 (National Institutes of Health) software obtained from http://rsb.info.nih.gov/ij/download.html.

High-resolution respirometry Mitochondrial respiration was determined in permeabilised WAT in an Oxygraph high-resolution respirometer chamber at $37^{\circ} \mathrm{C}$ (Oroboros Instruments, Innsbruck, Austria) as previously described $[22,23]$. ADP-stimulated respiration (state 3 ) was measured in the presence of pyruvate $(5 \mathrm{mmol} / \mathrm{l})$, malate $(5 \mathrm{mmol} / \mathrm{l})$ and $\operatorname{ADP}(5 \mathrm{mmol} / \mathrm{l})$ or malate $(5 \mathrm{mmol} / \mathrm{l})$, L-carnitine $(5 \mathrm{mmol} / \mathrm{l})$, palmitoyl-CoA $(50 \mu \mathrm{mol} / \mathrm{l})$ and ADP $(5 \mathrm{mmol} / \mathrm{l})$. Oligomycin-inhibited respiration (state 40) was determined in the presence of the above substrates with the addition of oligomycin $(6 \mu \mathrm{g} / \mathrm{ml})$. Cytochrome $c(10 \mu \mathrm{mol} / \mathrm{l})$ was added to the chamber to check the integrity of the outer mitochondrial membrane [24].

ROS measurements Mitochondrial $\mathrm{H}_{2} \mathrm{O}_{2}$ emissions were determined as previously described for skeletal muscle $[18,21]$. Briefly, $\sim 5 \mathrm{mg}$ (wet weight) finely minced adipose tissue was loaded into a cuvette containing Amplex Red (Invitrogen, Waltham, MA, USA), horseradish peroxidase $(1 \mathrm{U} / \mathrm{ml})$, digitonin $(10 \mu \mathrm{g} / \mathrm{ml})$ and oligomycin $(6.7 \mu \mathrm{g} / \mathrm{ml}$ ) in Buffer $\mathrm{Z}$ (K-MES $105 \mathrm{mmol} / \mathrm{l}, \mathrm{KCl}$ $30 \mathrm{mmol} / \mathrm{l}$, EGTA $1 \mathrm{mmol} / \mathrm{l}, \mathrm{K}_{2} \mathrm{HPO}_{4} 10 \mathrm{mmol} / \mathrm{l}, \mathrm{MgCl}_{2}$ $5 \mathrm{mmol} / \mathrm{l}$, glutamate $5 \mu \mathrm{mol} / \mathrm{l}$, malate $5 \mu \mathrm{mol} / \mathrm{l}$ and BSA $0.5 \%, \mathrm{pH}$ 7.4). Mitochondrial $\mathrm{H}_{2} \mathrm{O}_{2}$ emission rates were determined in the presences of DNP $(166.7 \mu \mathrm{mol} / \mathrm{l})$, ADP $(5 \mathrm{mmol} / \mathrm{l})$, rotenone $(6.7 \mu \mathrm{mol} / \mathrm{l})$, malonate $(6.7 \mathrm{mmol} / \mathrm{l})$ and/or with the addition of pyruvate + malate $(5 \mathrm{mmol} / \mathrm{l})$, succinate $(10 \mathrm{mmol} / \mathrm{l})$, glycerol 3-phosphate (G3P; $5 \mathrm{mmol} / \mathrm{l})$ or lipids; L-carnitine $(5 \mathrm{mmol} / \mathrm{l})+$ palmitoylCoA $(50 \mu \mathrm{mol} / \mathrm{l})$. A standard curve was made for each substrate with known concentrations of $\mathrm{H}_{2} \mathrm{O}_{2}$.

Quantitative real-time PCR RNA isolation and analysis was performed as previously described [25]. See the electronic supplementary material (ESM) for further details.

Statistics Results are expressed as mean \pm SEM and were analysed by Student's $t$ test or two-way repeated measures ANOVA followed by Bonferroni's post-hoc test where appropriate. Significance was set at $p<0.05$. All graphs and statistical analyses were performed using Prism 5.0 (GraphPad Software, La Jolla, CA, USA).

\section{Results}

HF feeding for 1 week induces WAT insulin resistance As anticipated, 1 week of HFD resulted in greater body weight and adipose tissue weight compared with chow-fed controls (Table 1), as well as insulin resistance at the whole-body level (AUC 1.16-fold, $p<0.001$ ) in awake mice (Fig. 1a). Although blood glucose levels were similar between HFD and chow mice (Table 1), serum insulin levels and HOMA-IR were 1.63-fold (Table 1, $p<0.05$ ) and 1.90-fold (Fig. 1b, $p<0.05$ ) higher with the HFD, respectively. Moreover, the ability of an intravenous insulin injection (15 $\mathrm{min}$ ) to decrease blood glucose levels in anaesthetised animals was severely blunted following the HFD (Fig. 1c). Interestingly, skeletal muscle insulin signalling was preserved after 1 week of the HFD (Fig. 1d). These results are consistent with previous studies showing skeletal muscle insulin resistance does not develop after only 1 week of HFD [26, 27].

Basal HSL phosphorylation, a surrogate marker of WAT lipolysis that is attenuated by insulin signalling, was increased by $\sim 1.60$-fold with the HFD (ESM Fig. 1a), while HFD resulted in a notable reduction in insulin-induced Ser-473 and Thr-308 Akt phosphorylation, particularly in the epididymal depot (Fig. 1e, f). Taken together, 1 week of HFD resulted in WAT-specific insulin resistance.

Overt WAT inflammation is not apparent after 1 week of HFD To evaluate potential causes of this rapid onset of dietinduced insulin resistance in WAT, we examined WAT inflammation and mitochondrial dysfunction. Histological examination showed no significant change in adipocyte cell size at 1 week on an HFD in either depot (Fig. 2a, b). In addition, F4/80 staining, a marker of macrophage infiltration, was similar between the two diets (Fig. 2c), suggesting no overt macrophage infiltration with short-term HF feeding. In agreement, the protein content of MCP-1, was similar between mice fed chow and those receiving the HFD (Fig. 2d). We also

Table 1 Body weight, adipose tissue weight and serum values after 1 week of dietary intervention

\begin{tabular}{lll}
\hline Variable & Chow & HFD \\
\hline Body weight $(\mathrm{g})$ & $35.4 \pm 1.0$ & $40.6 \pm 0.7^{* *}$ \\
Epididymal adipose tissue weight $(\mathrm{mg})$ & $1.68 \pm 0.15$ & $2.25 \pm 0.09^{*}$ \\
Inguinal adipose tissue weight $(\mathrm{mg})$ & $1.00 \pm 0.10$ & $1.33 \pm 0.05^{*}$ \\
Insulin (pmol/l) & $170.8 \pm 21.3$ & $279.1 \pm 31.4^{*}$ \\
Glucose (mmol/l) & $9.3 \pm 0.5$ & $10.6 \pm 0.8$ \\
NEFA (mmol/l) & $0.611 \pm 0.026$ & $0.621 \pm 0.046$ \\
Glycerol (mmol/l) & $0.631 \pm 0.068$ & $0.776 \pm 0.054^{\dagger}$ \\
\hline
\end{tabular}

Blood glucose and serum values were taken after a $2 \mathrm{~h}$ fast from anaesthetised animals. ${ }^{*} p<0.05, * * p<0.01$ and ${ }^{\dagger} p=0.06$ vs chow-fed mice 

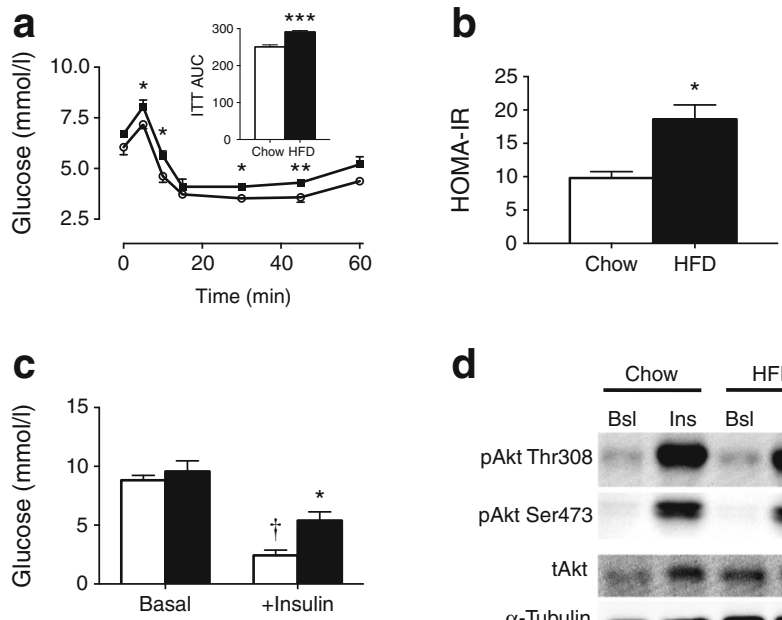

d
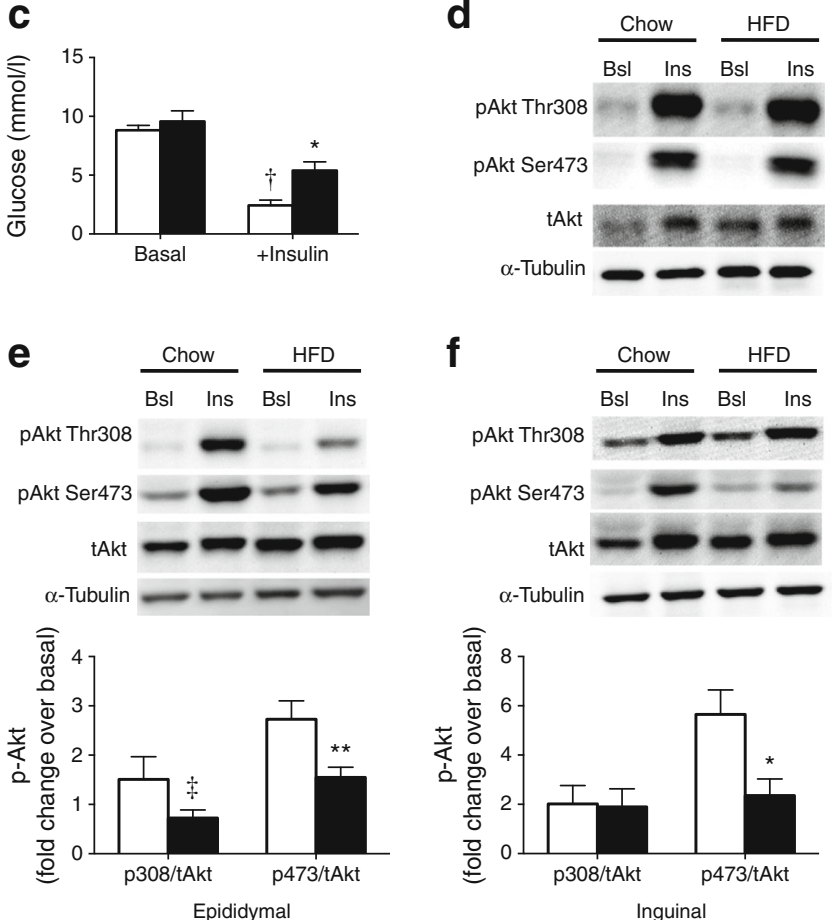

f
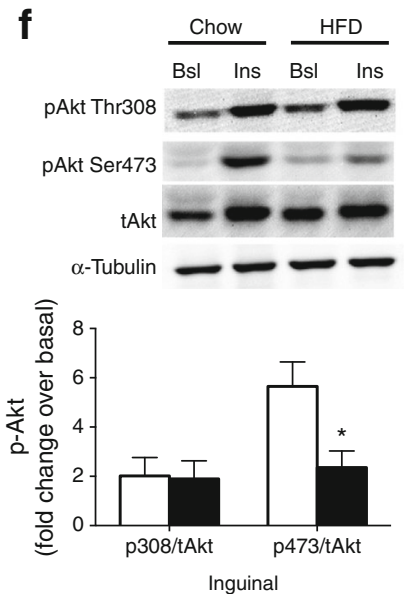

Fig. 1 An HFD for 1 week induces whole-body and WAT insulin resistance. (a) ITT and calculated AUC. (b) HOMA-IR in $2 \mathrm{~h}$ fasted mice. (c) Glucose levels before (Basal) and $15 \mathrm{~min}$ after an insulin injection (+Insulin). (d) Representative blots from muscle under basal and insulin-stimulated conditions. (e) Epididymal and (f) inguinal insulin-stimulated phosphorylated Akt fold change over basal. White circles/bars, chow-fed mice; black squares/bars, HFD-fed mice. ${ }^{*} p<0.05,{ }^{* *} p<0.01$, $* * * p<0.001$ and ${ }^{*} p=0.07$ vs chow-fed mice; ${ }^{\dagger} p<0.05$ vs basal. Bsl, basal; Ins, insulin-stimulated; t, total

observed no difference between the two diet groups for the metabolic and inflammatory signalling marker ERK1/2 (Fig. 2e, f). Meanwhile, P38-MAPK, which is activated by a plethora of proinflammatory and stress stimuli [28], remained unchanged after 1 week of HFD (ESM Fig. 1b). In contrast, examining the oxidative stress proinflammatory signalling marker JNK revealed increased phosphorylated JNK1 levels in the epididymal depot (Fig. 2g, h). With the exception of JNK, these data, when combined, suggest the absence of a strong inflammatory signal.

Mitochondrial respiratory capacity is not impaired with shortterm HFD As we excluded apparent macrophage infiltration as a putative mechanism causing short-term WAT insulin resistance, we next evaluated if defects within mitochondrial

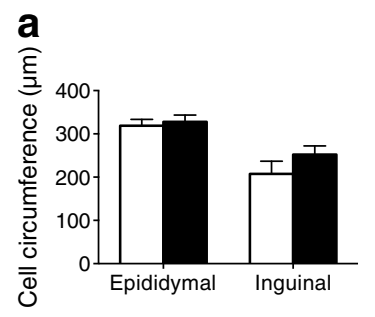

b

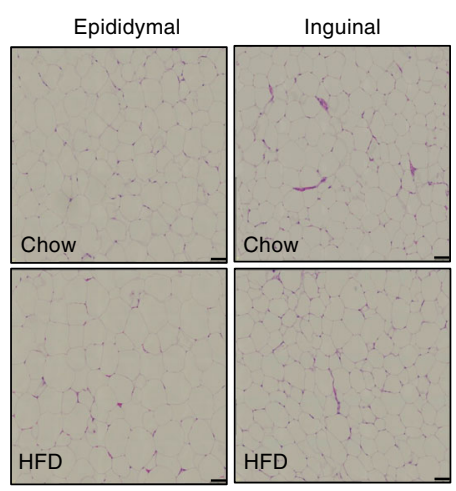

C
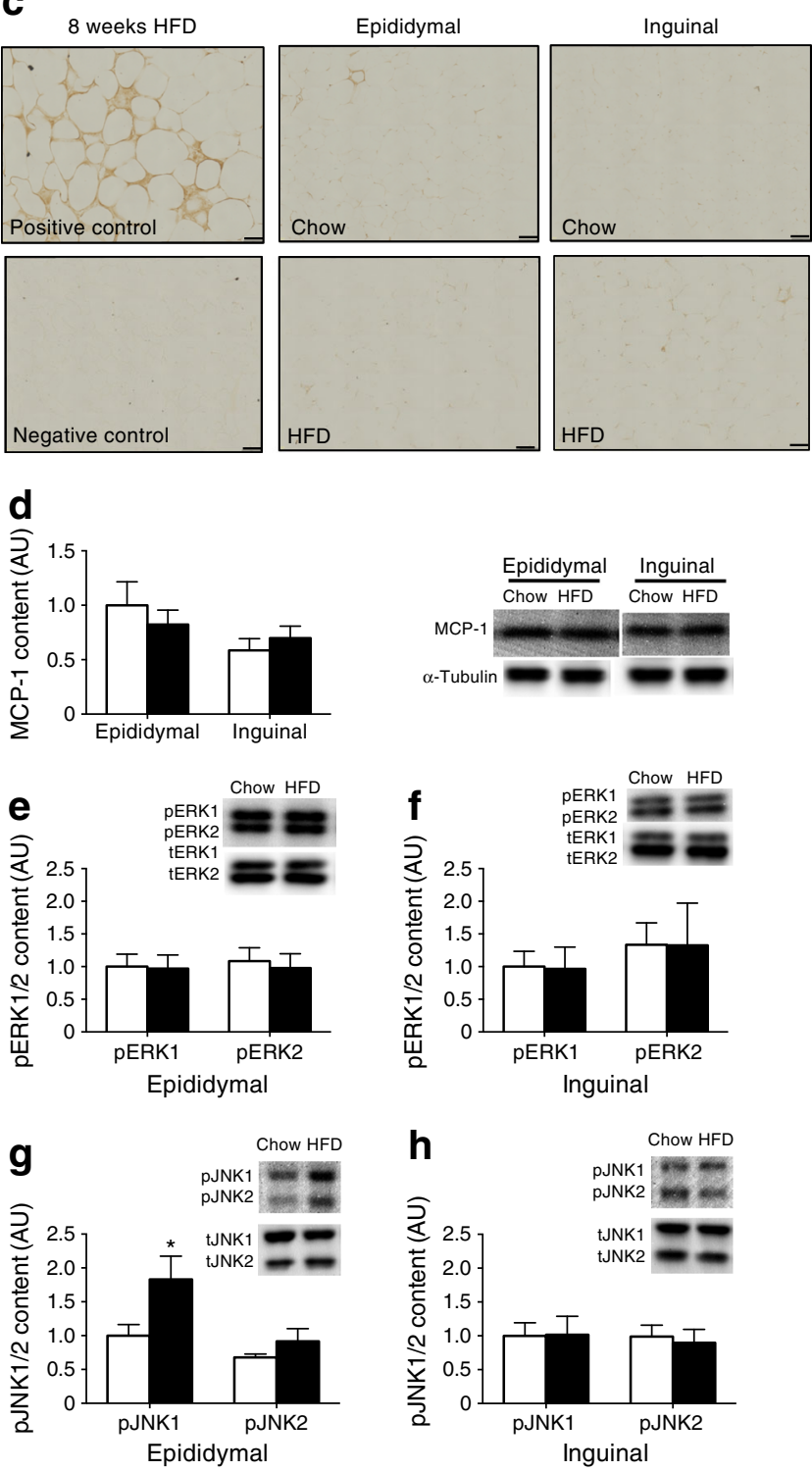

content and function are a potential mechanism of action. The protein content of various electron transport chain subunits was not different in either epididymal (Fig. 3a) or inguinal (Fig. 3b) depots following the HFD. Furthermore, 
4 Fig. 2 Short-term HFD increases JNK inflammatory response. (a) Adipocyte cell circumference. Sections stained with (b) haematoxylin and eosin and (c) F4/80, imaged at $\times 40$ magnification. Scale bars, $70 \mu \mathrm{m}$. Epididymal tissue from an 8 week HFD mouse served as a control in the presence (positive control) or absence (negative control) of primary antibody. (d) MCP-1, (e, f) ERK1/2 and (g, h) JNK1/2 content in WAT depots. Representative phosphorylated and total ERK1/2 and JNK1/2 blots. White bars, chow-fed mice; black bars, HFD-fed mice. ${ }^{*} p<0.05$ vs chow-fed mice. AU, arbitrary units/ $\mu$ g protein; $\mathrm{t}$, total

maximal DNP-stimulated mitochondrial respiratory function (data not shown) and ADP-stimulated (state 3) respiration supported by pyruvate (Fig. 3d, f) and lipids (Fig. 3e, g) as substrates were not altered by HFD. Leak respiration (state 4) occurs through uncoupling ATP synthesis from oxidative phosphorylation. Recently, ANT2-mediated uncoupling of mitochondria has been proposed to result in hypoxia, inflammation and WAT insulin resistance [3]. However, in the present study oligomycin-uncoupled respiration (state 4o) was similar between chow- and HF-fed mice for each substrate tested (Fig. 3d-g). Therefore, mitochondrial respiratory dysfunction is not a causal factor regulating JNK phosphorylation or insulin resistance within adipose tissue.

Insulin-resistant epididymal tissue is associated with greater degree of lipid peroxidation Oxidative stress is known to activate JNK signalling, and therefore we next considered alterations in the redox environment as a potential cause of WAT insulin resistance. HFD increased the lipid peroxidation adduct, 4-HNE, in epididymal tissue ( $\sim 1.70$-fold, $p<0.05$, Fig. $4 \mathrm{a}$ ). In contrast, 4-HNE content was similar between chow- and HF-fed mice in the inguinal depot (Fig. 4a), and no difference was observed for protein carbonylation (Fig. 4b) in either depot. Similarly, levels of mitochondrial SOD2 a
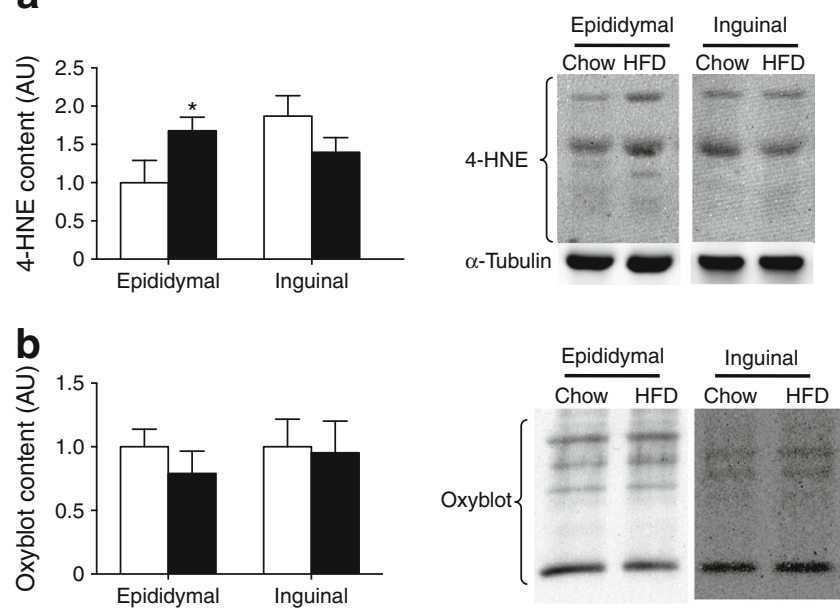

C

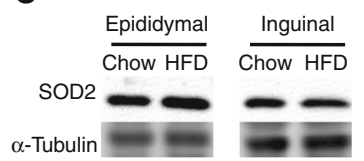

d
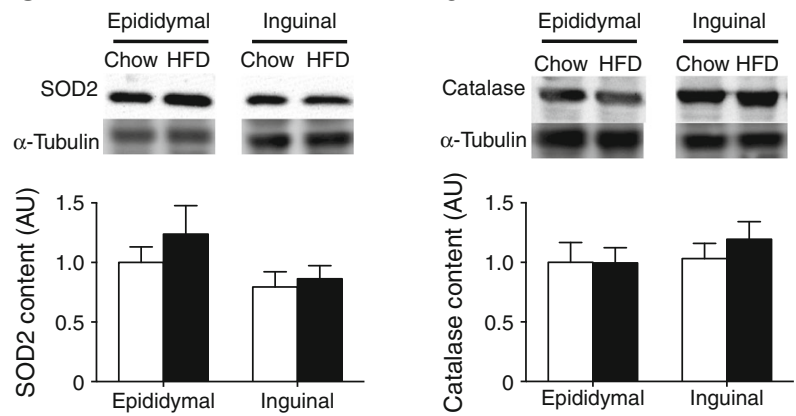

Fig. 4 Markers of oxidative stress and antioxidant content. (a) 4-HNE, (b) protein carbonylation, (c) SOD2 and (d) catalase content in epididymal and inguinal depots. White bars, chow-fed mice; black bars, HFD-fed mice. ${ }^{*} p<0.05$ vs chow-fed mice. AU, arbitrary units $/ \mu \mathrm{g}$ protein

(Fig. 4c) and catalase (Fig. 4d) were comparable between chow- and HF-fed mice in both depots. Therefore, short-
Fig. 3 HFD does not affect WAT mitochondrial content or respiration. Mitochondrial content in (a) epididymal and (b) inguinal depots measured by an OXPHOS antibody and (c) representative blots. ADPstimulated (state 3 ) and oligomycin-inhibited (state 4o) mitochondrial respiration in $(\mathbf{d}, \mathbf{e})$ epididymal and (f, $\mathbf{g}$ ) inguinal depots. White bars, chow-fed mice; black bars, HFD-fed mice. $\alpha \mathrm{T}, \alpha$-tubulin; $J \mathrm{O}_{2}$, oxygen flux
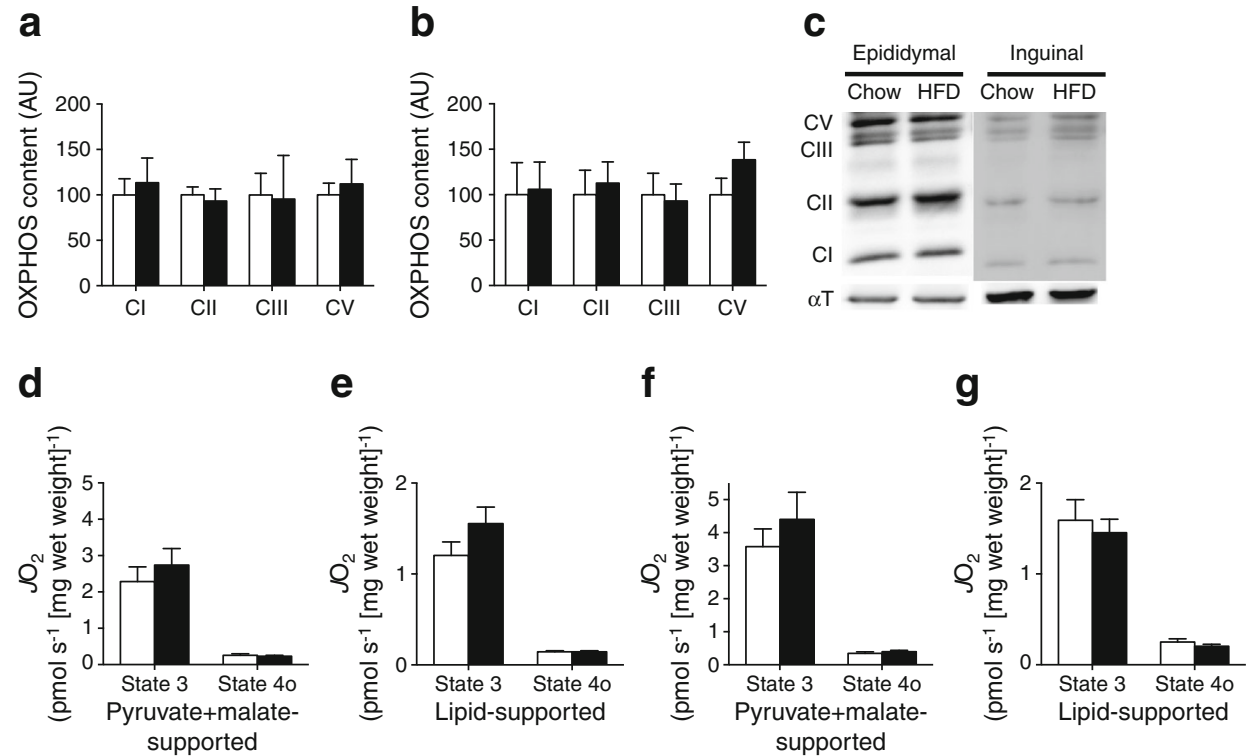
a
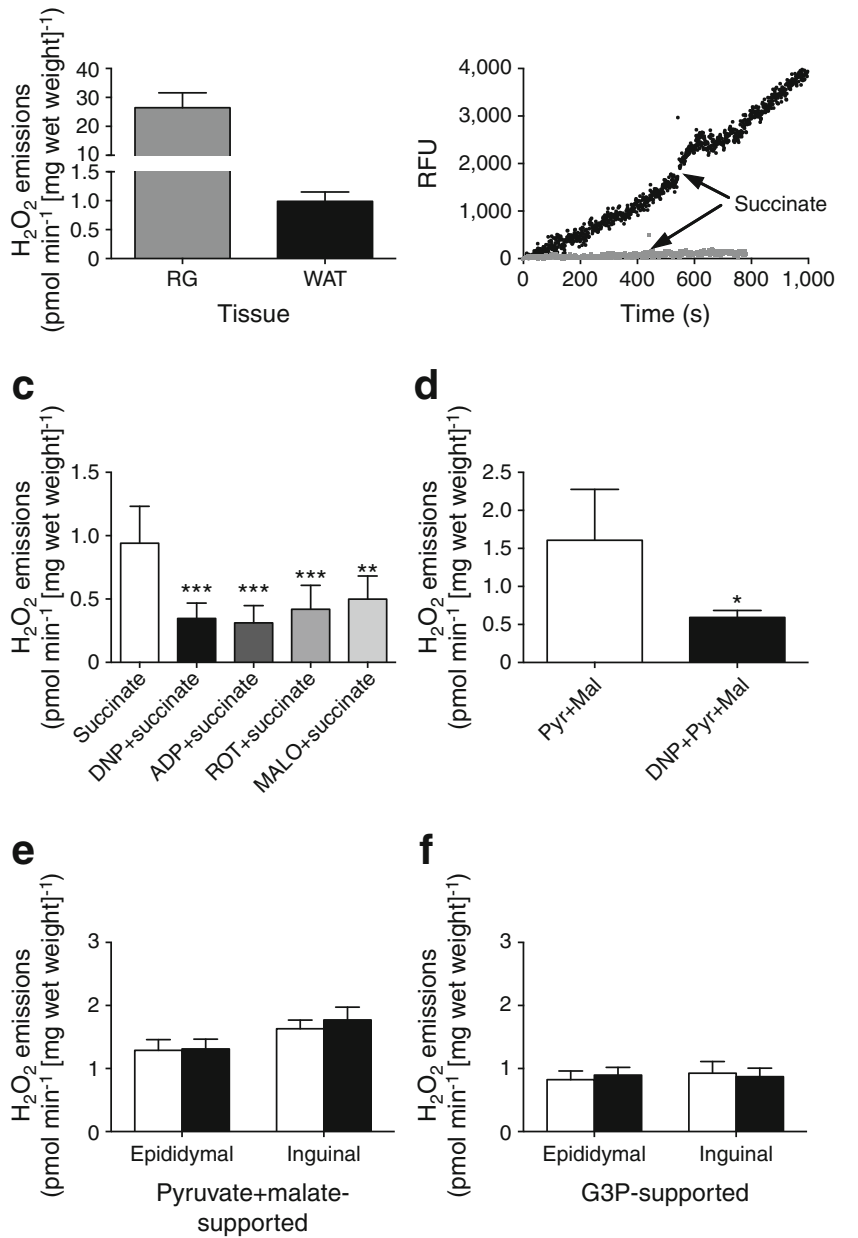

g

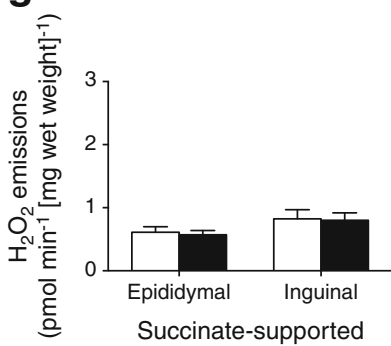

b

d

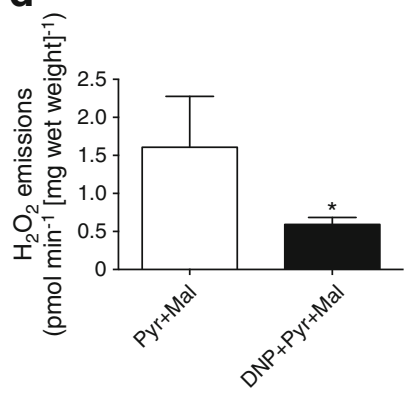

f

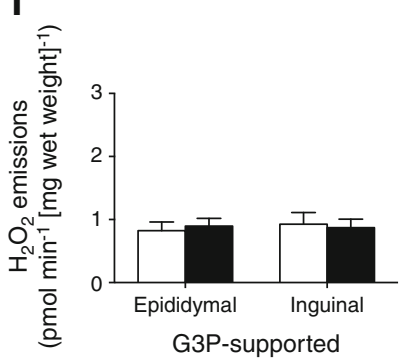

h

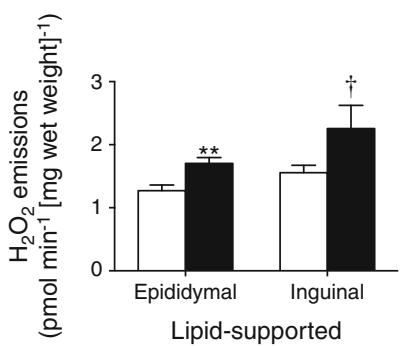

Fig. 5 Lipid-supported mitochondrial ROS emission rates are increased with HF feeding. (a) Succinate-supported mitochondrial ROS emissions from red gastrocnemius muscle permeabilised fibres and WAT. (b) Representative trace of permeabilised WAT matched for wet weight without (black line) and with (grey line) the uncoupler DNP. The addition of succinate is indicated with an arrow. Corrected (c) succinate-supported or (d) pyruvate-supported mitochondrial ROS emissions alone or in the presence of uncouplers or inhibitors; ${ }^{*} p<0.05, * * p<0.01$ and $* * * p<0.001$ vs substrate alone. (e-h) Substrate-supported mitochondrial ROS emission rates. White bars, chow-fed mice; black bars, HFD-fed mice. ${ }^{* *} p<0.01$ and ${ }^{\dagger} p=0.08$ vs chow-fed mice. Mal, malate; MALO, malonate; ROT, rotenone; Pyr, pyruvate; RFU, relative fluorescence units; RG, red gastrocnemius

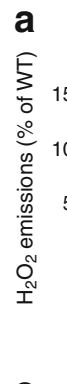

C

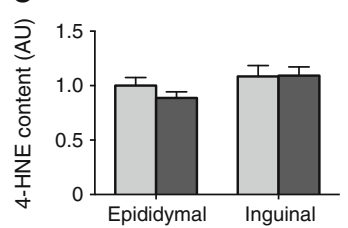

b

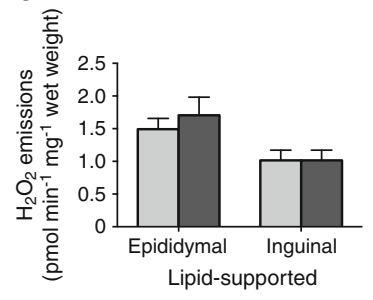

d
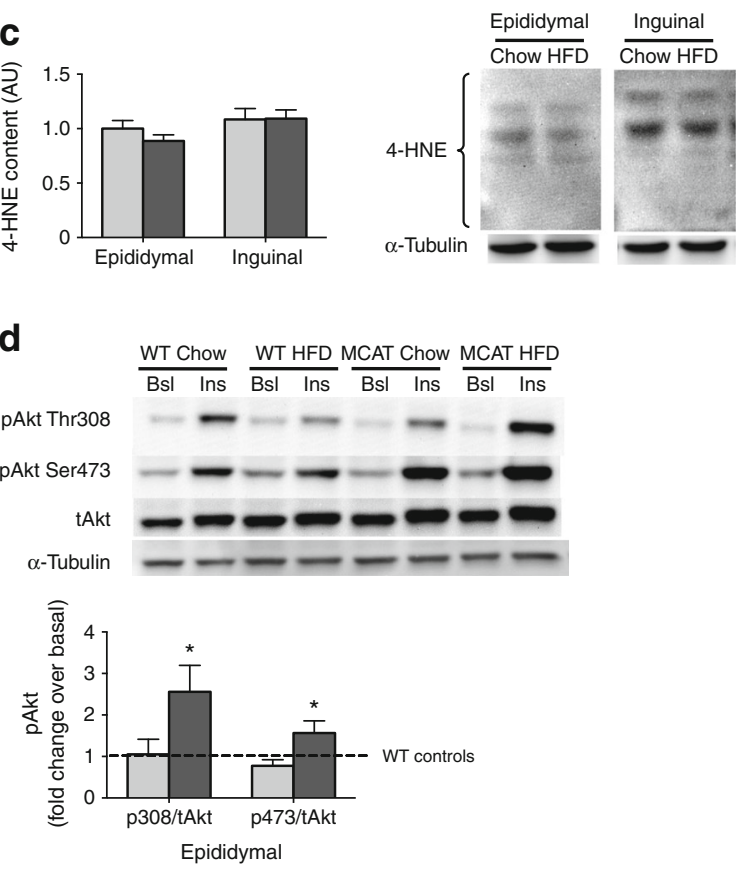

e WT Chow WT HFD MCAT Chow MCAT HFD
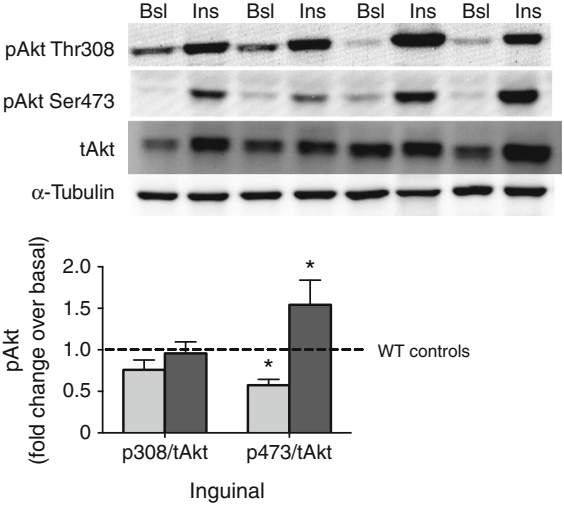

f

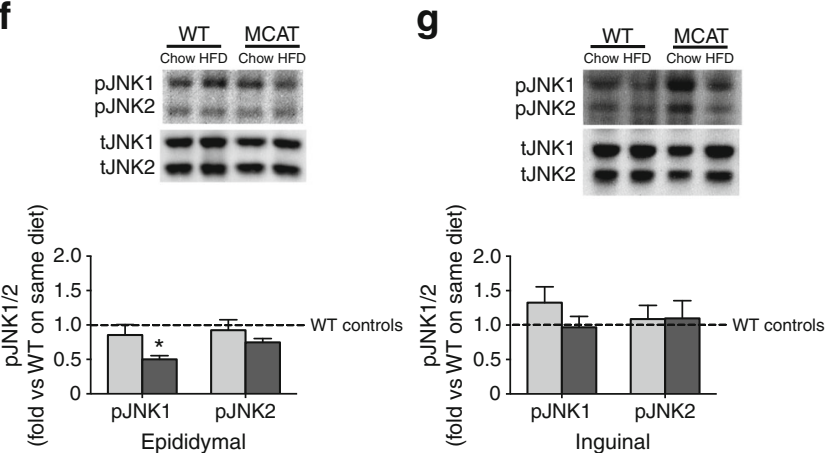


Fig. 6 Preserved mitochondrial ROS emission rates and insulin signalling in MCAT mice on an HFD. (a) Relative succinate and lipidsupported mitochondrial ROS emission from WT (white bars) and MCAT (light grey bars) mice. (b) Lipid-supported mitochondrial ROS emission rates in MCAT mice. (c) 4-HNE content and representative blots. (d) Epididymal and (e) inguinal insulin-stimulated phosphorylated Akt fold change over basal condition relative to WT mice on the same diet. Representative blots shown for basal and insulin-stimulated conditions, $* p<0.05$ vs WT on same diet. JNK $1 / 2$ content relative to WT mice on the same diet in (f) epididymal and (g) inguinal depots and representative phosphorylated and total JNK blots. For (b-g) light grey bar and dark grey bars represent MCAT chow- and HF-fed mice, respectively. AU, arbitrary units/ $\mu \mathrm{g}$ protein; Bsl, basal; Ins, insulin-treated; $t$, total

term HFD-induced insulin resistance is associated with a lipid-specific oxidative stress marker, particularly in epididymal tissue.

Short-term HFD increases lipid-supported mitochondrial ROS emissions Next, we sought to determine mitochondrial ROS emission rates in WAT as a source of the observed oxidative stress. Therefore, to measure mitochondrial ROS emissions in WAT, we adopted a protocol previously established for permeabilised skeletal muscle fibres [18, 21]. While WAT mitochondrial $\mathrm{H}_{2} \mathrm{O}_{2}$ emission rates are lower than skeletal muscle fibres (Fig. 5a), owing to differences in mitochondrial content between the two tissues, WAT mitochondrial ROS emissions react similarly to skeletal muscle. Specifically, in WAT, mitochondrial $\mathrm{H}_{2} \mathrm{O}_{2}$ emission rates are increased with excess substrates (e.g. succinate addition) and are dissipated by $\sim 50-70 \%$ in the presence of uncouplers (DNP and exogenous ADP) or complex inhibitors (Fig. 5b-d). Therefore, we further investigated mitochondrial ROS emission rates using several substrates to gain insight into the different electron 'leakage points'. Mitochondrial ROS emission rates were similar between chow- and HFD-fed mice when supported by pyruvate and malate (Fig. 5e), G3P (Fig. 5f) or succinate (Fig. 5g) in epididymal and inguinal depots. However, mitochondrial ROS emissions increased by 1.33 -fold $(p<0.01)$ and 1.15 -fold $(p=0.08)$ in HFD mice with a lipid-supported substrate in epididymal and inguinal depots, respectively (Fig. 5h). Therefore, HFD-induced adipose tissue insulin resistance may be a consequence of increased lipid-supported mitochondrial ROS emissions.

Transgenic mice with decreased mitochondrial ROS emissions are protected from diet-induced insulin resistance We next aimed to determine if increased lipid-supported mitochondrial ROS is a causal factor in short-term diet-induced insulin resistance. To accomplish this we used the MCAT mouse model to determine if decreasing mitochondrial ROS prevented the observed acute HFD attenuation in WAT insulin sensitivity. MCAT mice express the human catalase gene within mitochondria and are a model of increased mitochondrial antioxidant enzyme capacity [17]. The original characterisation of these animals did not determine the presence of the transgene in WAT. Here, we demonstrate that human catalase is expressed in WAT of MCAT mice to a similar extent as endogenous mouse catalase (ESM Fig. 2a). Moreover, mitochondrial ROS emissions are reduced by $\sim 40 \%$ in WAT from MCAT compared with WT mice (Fig. 6a), validating the use of MCAT mice as a model to investigate the mechanistic link between increased WAT ROS and insulin resistance.

When placed on an HFD, MCAT mice were protected from increased lipid-supported mitochondrial ROS emissions (Fig. 6b); the lipid oxidative stress marker 4-HNE was not increased (Fig. 6c), contrary to WT HFD mice (Fig. 4a). Furthermore, the consumption of an HFD did not increase mitochondrial succinate-, pyruvate- or G3P-supported ROS emission rates in MCAT mice (data not shown). In addition, whole-body insulin tolerance (ESM Fig. 2b) and both epididymal (Fig. 6d) and inguinal (Fig. 6e) insulin signalling were preserved in MCAT HFD mice.

Finally, JNK phosphorylation was similar between chow and HFD MCAT mice in both depots (Fig. 6f, g), indicating a subdued inflammatory response. Taken together, MCAT mice are protected from the observed HFD-induced activation of JNK signalling and the attenuation of WAT insulin signalling, providing proof of principle that increased mitochondrial ROS has a causal role in the initiation of WAT insulin resistance.

\section{Discussion}

In the present study we demonstrated that 1 week of HF feeding increased lipid-supported mitochondrial ROS emissions, lipid-specific oxidative stress and induced insulin resistance in WAT. Moreover, we provide evidence that increased mitochondrial antioxidant capacity, and the associated decreased mitochondrial ROS emission, prevents increased markers of oxidative stress, inflammation and attenuations in insulin signalling in WAT on HF feeding. Taken together, the current data implicate increased mitochondrial ROS emission as an early mechanism inducing WAT insulin resistance.

While low-grade inflammation increases oxidative stress in the obese state [29], the temporal cause-and-effect relationship between inflammation, oxidative stress and insulin resistance remains poorly defined. In the present study, F4/80 immunohistochemical staining, indicating blatant adipose tissue inflammation, was minimal after 1 week of HFD. However, the oxidative-stress-mediated inflammatory signalling response was increased in the visceral depot. This is in line with other studies showing that short-term HFD induces inflammatory response signals $[6,30,31]$. As chronic HFD is associated with increased oxidative stress from excessive 
mitochondrial ROS emissions in muscle [18, 32], we sought to evaluate the induction of oxidative stress from mitochondrial ROS emissions in WAT after 1 week on an HFD. Unlike JNK phosphorylation, increased lipid-supported mitochondrial $\mathrm{H}_{2} \mathrm{O}_{2}$ emission was associated with the development of insulin resistance in both WAT depots studied, suggesting mitochondrial ROS may contribute directly to the establishment of insulin resistance within WAT.

We also measured two markers of oxidative stress, 4-HNE and total protein carbonylation. An aldehyde product of lipid peroxidation, 4-HNE is one of the most abundant aldehydes found in adipose tissue [33]. Although protein carbonylation was similar between the diet groups, after 1 week of HF feeding we observed a significant increase in the visceral adipose depot 4-HNE content, with no change in the subcutaneous depot. This is in agreement with Long et al who also showed depot-specific effects in 4-HNE accumulation: 9 weeks of HFD increased 4-HNE content five-fold in epididymal tissue, but 4-HNE was decreased in the subcutaneous depot [33]. It has been shown that 4-HNE can directly interfere with insulin signalling, as exogenous exposure of cultured adipocytes to 4HNE results in reduced IRS-1 content and phosphorylation [34]. More recently, 4-HNE was shown to inhibit Akt activity and insulin signalling downstream of Akt in hepatocytes [35]; however, this has not yet been evaluated in adipocytes. Nonetheless, it is tempting to speculate that, in the current study, the increased 4-HNE levels within epididymal adipose tissue, in addition to the possible direct effects of $\mathrm{H}_{2} \mathrm{O}_{2}$, contributed to the exaggerated impairment in insulin signalling observed in this depot.

To explore the underlying mechanism causing rapid HFDinduced insulin resistance, we used the MCAT mouse, a model of upregulated mitochondrial antioxidant enzyme capacity. Catalase is the enzyme that converts reactive $\mathrm{H}_{2} \mathrm{O}_{2}$ into $\mathrm{H}_{2} \mathrm{O}$, and therefore MCAT mice can quench mitochondrial ROS emissions in situ, thus alleviating excessive ROS emissions and eliminating overt oxidative stress. This model has been previously employed to investigate the role of oxidative stress in age-related cardiomyopathy [17]. More recently, MCAT mice were shown to be protected against age- [19] and HFD- [18] induced insulin resistance in skeletal muscle. Specifically, improved insulin sensitivity in MCAT mice was associated with blunted skeletal muscle mitochondrial ROS emissions and reduced oxidative stress [18, 19].

Here, we demonstrated that the human catalase gene is expressed in the WAT of MCAT mice, and is associated with attenuated mitochondrial ROS emission. Moreover, we showed that insulin signalling within WAT was preserved with 1 week of HFD in MCAT mice while lipid-supported mitochondrial ROS emissions, lipid peroxidation and inflammatory signalling were normalised, suggesting excess ROS is the principal mechanism in adipose tissue dysfunction. These findings are in agreement with those of Pires et al who demonstrated that ROS sequestering through the administration of a SOD mimetic reduced WAT inflammation with 5 weeks of HFD [36]. Therefore, mitochondrial ROS emissions are a potential early causal factor in diet-induced insulin resistance in WAT. More recently, Asterholm et al demonstrated the requirement of an acute inflammatory response to facilitate adipogenesis and remodelling under an HF challenge [37]. In the context of the current data, HFD-induced increases in mitochondrial ROS are implicated in these events, though this remains to be shown directly. In this manner, mitochondrial ROS may represent a sensitive barometer of excess fuel in WAT and induce gene transcriptional programmes that result in adipogenesis.

To our knowledge the present study is the first to examine mitochondrial ROS emission in permeabilised WAT in the context of diet-induced insulin resistance. While previous studies have evaluated ROS emissions in cultured cell systems or isolated adipocytes [38-40], the advantage of permeabilised WAT over these traditional methods is the ability to assess adipocyte function within its cellular milieu. Approximately $25 \%$ of WAT is composed of adipocytes, the remaining cell population is made up of the stromal vascular cells (SVC), including macrophages [41]. SVC are integral members of WAT contributing to function of the organ, and while our method does not discriminate between the sources of mitochondrion (for example, macrophage vs adipocyte), we can evaluate how WAT, as a whole tissue, responds to various substrates. Importantly, the MCAT mice clearly delineate a functional role for mitochondrially derived ROS in the induction of insulin resistance within WAT.

We examined four key electron 'leakage' sites of mitochondrial ROS production through the use of different substrates. Mitochondrial ROS emissions from complex I and III, caused by electron leakage, increase on building of membrane potential, which can occur under conditions of high substrate availability. Meanwhile, mitochondrial ROS emissions derived from G3P dehydrogenase (GPDH) or the electron transfer flavoprotein (ETF) have been proposed to be independent of membrane potential $[42,43]$. In brown adipose tissue, GPDH is highly prone to electron leakage, resulting in high rates of ROS emissions [44]. However, in WAT we demonstrated that G3P-supported emission rates were fairly low, possibly because of differences in mitochondrial content between brown adipose tissue and WAT. Interestingly, contrary to skeletal muscle, in WAT the rates of succinate-supported ROS emissions were lower than those of pyruvate- or lipid-supported mitochondrial ROS emissions. This is likely due to forward electron flow owing to residual endogenous substrates not washed away in our permeabilised preparation. While we did not fully explore ROS emission topology in the present study, we observed the highest emission rates with a lipid substrate. In skeletal muscle, Seifert et al demonstrated that ROS production at the ETF occurs at a wide range of 
physiological concentrations [43], consequentially leading to adverse effects, especially in the context of high lipid availability. In this regard, 1 week of HFD did not change pyruvate-, succinate- or G3P-supported mitochondrial ROS rates, yet lipid-supported ROS rates were significantly elevated in epididymal tissue, with a strong trend to increase in the inguinal depot. Recently, it was suggested that acute HFD-induced mitochondrial uncoupling, and the resulting increase in WAT oxygen consumption, can propagate a hypoxic environment leading to insulin-resistant conditions [3]. While mitochondrial uncoupling would dissipate ROS emissions, we seemingly observed contrary findings that HFD promoted mitochondrial ROS emissions and increased oxidative stress. Furthermore, mitochondrial ROS emissions were specific to a lipidsupported substrate, which can generate ROS emissions independent of membrane potential [43]. Taken together, these data suggest that lipid-supported mitochondrial ROS emission increased oxidative stress resulting in insulin resistance, specifically within the visceral depot.

In summary, we have assessed the role of diet-induced oxidative stress and mitochondrial ROS emissions in WAT after 1 week of HF feeding. We found that marked lipidsupported mitochondrial ROS emissions were associated with an increase in lipid peroxidation, elevated inflammatory signalling and impaired insulin signalling, which were abolished in MCAT mice. Therefore, we propose that elevated mitochondrial ROS emission observed after 1 week of a HFD is a causal factor of WAT insulin resistance which contributes to altered whole-body glucose homeostasis.

Acknowledgements We would like to thank D. C. Wright (University of Guelph) for generously providing some of the antibodies used in this study.

Funding This work was funded by the Natural Sciences and Engineering Research Council of Canada (NSERC; GPH). Infrastructure was purchased with the assistance of the Canadian Foundation for Innovation $(\mathrm{GPH})$ as well as the Ontario Research Fund (GPH).

Duality of interest The authors declare that there is no duality of interest associated with this manuscript.

Contribution statement SP designed the study, researched and interpreted data, and wrote the manuscript. AL and JR-M researched data, interpreted data and edited the manuscript. GPH designed the study, contributed to writing the manuscript and is the guarantor of this study. All authors have approved the final version of the manuscript.

\section{References}

1. Bjorntorp P, Berchtold P, Holm J, Larsson B (1971) The glucose uptake of human adipose tissue in obesity. Eur J Clin Investig $1: 480-485$

2. Marin P, Rebuffe-Scrive M, Smith U, Bjorntorp P (1987) Glucose uptake in human adipose tissue. Metabolism 36:1154-1160
3. Lee YS, Kim JW, Osborne $\mathrm{O}$ et al (2014) Increased adipocyte $\mathrm{O} 2$ consumption triggers HIF-1alpha, causing inflammation and insulin resistance in obesity. Cell 157:1339-1352

4. Lee YS, Li P, Huh JY et al (2011) Inflammation is necessary for longterm but not short-term high-fat diet-induced insulin resistance. Diabetes 60:2474-2483

5. Williams LM, Campbell FM, Drew JE et al (2014) The development of diet-induced obesity and glucose intolerance in C57BL/6 mice on a high-fat diet consists of distinct phases. PLoS One 9:e106159

6. Cummins TD, Holden CR, Sansbury BE et al (2014) Metabolic remodeling of white adipose tissue in obesity. Am J Physiol Endocrinol Metab 307(307):E262-E277

7. Ahmadian M, Duncan RE, Sul HS (2009) The skinny on fat: lipolysis and fatty acid utilization in adipocytes. Trends Endocrinol Metab 20:424-428

8. Xu H, Barnes GT, Yang Q et al (2003) Chronic inflammation in fat plays a crucial role in the development of obesity-related insulin resistance. J Clin Invest 112:1821-1830

9. Hotamisligil GS, Shargill NS, Spiegelman BM (1993) Adipose expression of tumor necrosis factor-alpha: direct role in obesity-linked insulin resistance. Science 259:87-91

10. Furukawa S, Fujita T, Shimabukuro M et al (2004) Increased oxidative stress in obesity and its impact on metabolic syndrome. J Clin Invest 114:1752-1761

11. Chattopadhyay M, Khemka VK, Chatterjee G, Ganguly A, Mukhopadhyay S, Chakrabarti S (2015) Enhanced ROS production and oxidative damage in subcutaneous white adipose tissue mitochondria in obese and type 2 diabetes subjects. Mol Cell Biochem 399:95-103

12. Li B, Nolte LA, Ju JS et al (2000) Skeletal muscle respiratory uncoupling prevents diet-induced obesity and insulin resistance in mice. Nat Med 6:1115-1120

13. Bi P, Shan T, Liu W et al (2014) Inhibition of Notch signaling promotes browning of white adipose tissue and ameliorates obesity. Nat Med 20:911-918

14. Perry RJ, Kim T, Zhang XM et al (2013) Reversal of hypertriglyceridemia, fatty liver disease, and insulin resistance by a liver-targeted mitochondrial uncoupler. Cell Metab 18:740-748

15. Korshunov SS, Skulachev VP, Starkov AA (1997) High protonic potential actuates a mechanism of production of reactive oxygen species in mitochondria. FEBS Lett 416:15-18

16. Zorov DB, Filburn CR, Klotz LO, Zweier JL, Sollott SJ (2000) Reactive oxygen species (ROS)-induced ROS release: a new phenomenon accompanying induction of the mitochondrial permeability transition in cardiac myocytes. J Exp Med 192:1001-1014

17. Schriner SE, Linford NJ, Martin GM et al (2005) Extension of murine life span by overexpression of catalase targeted to mitochondria. Science 308:1909-1911

18. Anderson EJ, Lustig ME, Boyle KE et al (2009) Mitochondrial $\mathrm{H} 2 \mathrm{O} 2$ emission and cellular redox state link excess fat intake to insulin resistance in both rodents and humans. J Clin Invest 119:573-581

19. Lee HY, Choi CS, Birkenfeld AL et al (2010) Targeted expression of catalase to mitochondria prevents age-associated reductions in mitochondrial function and insulin resistance. Cell Metab 12:668-674

20. Jain SS, Snook LA, Glatz JF et al (2012) Munc18c provides stimulus-selective regulation of GLUT4 but not fatty acid transporter trafficking in skeletal muscle. FEBS Lett 586:2428-2435

21. Herbst EA, Paglialunga S, Gerling C et al (2014) Omega-3 supplementation alters mitochondrial membrane composition and respiration kinetics in human skeletal muscle. J Physiol 592:1341-1352

22. Beaudoin MS, Snook LA, Arkell AM, Simpson JA, Holloway GP, Wright DC (2013) Resveratrol supplementation improves white adipose tissue function in a depot-specific manner in Zucker diabetic fatty rats. Am J Physiol Regul Integr Comp Physiol 305:R542-R551 
23. Wan Z, Perry CG, Macdonald T, Chan CB, Holloway GP, Wright DC (2012) IL-6 is not necessary for the regulation of adipose tissue mitochondrial content. PLoS One 7:e51233

24. Perry CG, Kane DA, Lin CT et al (2011) Inhibiting myosin-ATPase reveals a dynamic range of mitochondrial respiratory control in skeletal muscle. Biochem J 437:215-222

25. Matravadia S, Martino VB, Sinclair D, Mutch DM, Holloway GP (2013) Exercise training increases the expression and nuclear localization of mRNA destabilizing proteins in skeletal muscle. Am J Physiol Regul Integr Comp Physiol 305:R822-R831

26. Winzell MS, Magnusson C, Ahren B (2007) Temporal and dietary fat content-dependent islet adaptation to high-fat feeding-induced glucose intolerance in mice. Metabolism 56:122-128

27. Turner N, Kowalski GM, Leslie SJ et al (2013) Distinct patterns of tissue-specific lipid accumulation during the induction of insulin resistance in mice by high-fat feeding. Diabetologia 56:1638-1648

28. Zarubin T, Han J (2005) Activation and signaling of the p38 MAP kinase pathway. Cell Res 15:11-18

29. Fernandez-Sanchez A, Madrigal-Santillan E, Bautista M et al (2011) Inflammation, oxidative stress, and obesity. Int J Mol Sci 12: 3117-3132

30. Wiedemann MS, Wueest S, Item F, Schoenle EJ, Konrad D (2013) Adipose tissue inflammation contributes to short-term high-fat dietinduced hepatic insulin resistance. Am J Physiol Endocrinol Metab 305:E388-E395

31. Ji Y, Sun S, Xia S, Yang L, Li X, Qi L (2012) Short term high fat diet challenge promotes alternative macrophage polarization in adipose tissue via natural killer T cells and interleukin-4. J Biol Chem 287: 24378-24386

32. Paglialunga S, van Bree B, Bosma M et al (2012) Targeting of mitochondrial reactive oxygen species production does not avert lipidinduced insulin resistance in muscle tissue from mice. Diabetologia 55:2759-2768

33. Long EK, Olson DM, Bernlohr DA (2013) High-fat diet induces changes in adipose tissue trans-4-oxo-2-nonenal and trans-4hydroxy-2-nonenal levels in a depot-specific manner. Free Radic Biol Med 63:390-398
34. Demozay D, Mas JC, Rocchi S, van Obberghen E (2008) FALDH reverses the deleterious action of oxidative stress induced by lipid peroxidation product 4-hydroxynonenal on insulin signaling in 3T3-L1 adipocytes. Diabetes 57:1216-1226

35. Shearn CT, Fritz KS, Reigan P, Petersen DR (2011) Modification of Akt2 by 4-hydroxynonenal inhibits insulin-dependent Akt signaling in HepG2 cells. Biochemistry 50:3984-3996

36. Pires KM, Ilkun O, Valente M, Boudina S (2013) Treatment with a SOD mimetic reduces visceral adiposity, adipocyte death, and adipose tissue inflammation in high fat-fed mice. Obesity (Silver Spring) 22:178-187

37. Asterholm IW, Toa C, Morley TS et al (2014) Adipocyte inflammation is essential for healthy adipose tissue expansion and remodeling. Cell Metab 20:14

38. Houstis N, Rosen ED, Lander ES (2006) Reactive oxygen species have a causal role in multiple forms of insulin resistance. Nature 440:944-948

39. Hahn WS, Kuzmicic J, Burrill JS et al (2014) Proinflammatory cytokines differentially regulate adipocyte mitochondrial metabolism, oxidative stress, and dynamics. Am J Physiol Endocrinol Metab 306: E1033-E1045

40. Tormos KV, Anso E, Hamanaka RB et al (2011) Mitochondrial complex III ROS regulate adipocyte differentiation. Cell Metab 14:537-544

41. Mraz M, Haluzik M (2014) The role of adipose tissue immune cells in obesity and low-grade inflammation. J Endocrinol 222: R113-R127

42. Miwa S, St-Pierre J, Partridge L, Brand MD (2003) Superoxide and hydrogen peroxide production by Drosophila mitochondria. Free Radic Biol Med 35:938-948

43. Seifert EL, Estey C, Xuan JY, Harper ME (2010) Electron transport chain-dependent and -independent mechanisms of mitochondrial $\mathrm{H} 2 \mathrm{O} 2$ emission during long-chain fatty acid oxidation. J Biol Chem 285:5748-5758

44. Vrbacky M, Drahota Z, Mracek T et al (2007) Respiratory chain components involved in the glycerophosphate dehydrogenasedependent ROS production by brown adipose tissue mitochondria. Biochim Biophys Acta 1767:989-997 